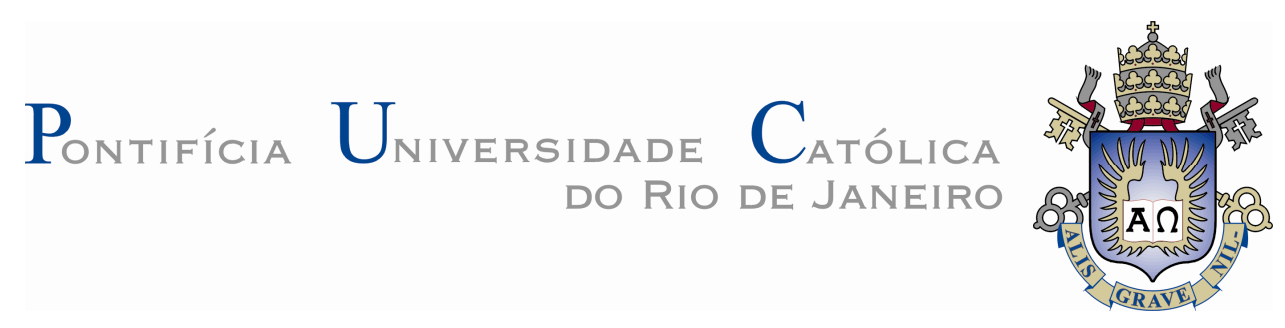

Fillipe Machado Pinto Napolitano

\title{
Uma Estratégia Baseada em Simulação para Validação de Modelos em i ${ }^{*}$
}

\section{Dissertação de Mestrado}

Dissertação apresentada ao Programa de PósGraduação em Informática da PUC-Rio como requisito parcial para obtenção do título de Mestre em Informática.

Orientador: Prof. Julio Cesar Sampaio do Prado Leite

Rio de Janeiro

Agosto de 2009 


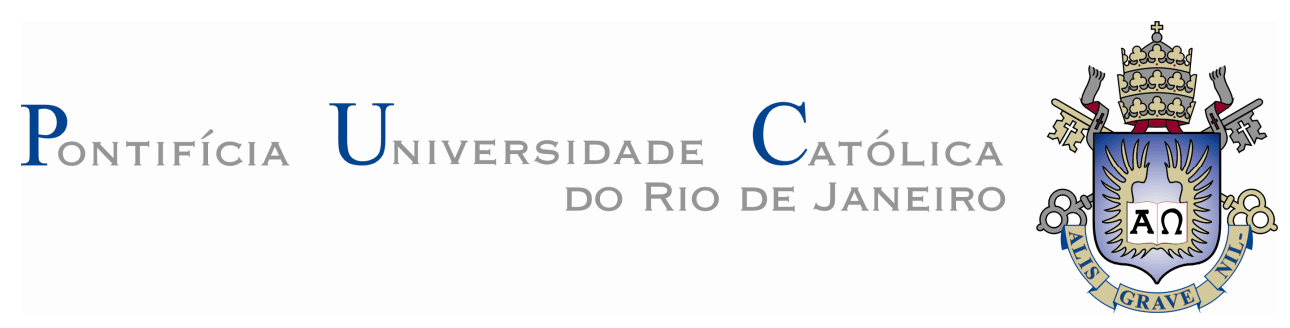

Fillipe Machado Pinto Napolitano

\section{Uma Estratégia Baseada em Simulação para Validação de Modelos em i*}

Dissertação apresentada como requisito parcial para obtenção do grau de Mestre pelo Programa de PósGraduação em Informática do Departamento de Informática do Centro Técnico e Científico da PUC-Rio. Aprovada pela Comissão Examinadora abaixo assinada.

Julio Cesar Sampaio do Prado Leite Orientador

Departamento de Informática - PUC-Rio

Marco Antônio Casanova

Departamento de Informática - PUC-Rio

Simone Diniz Junqueira Barbosa Departamento de Informática - PUC-Rio

José Eugênio Leal Coordenador Setorial do Centro Técnico Científico - PUC-Rio 
Todos os direitos reservados. É proibida a reprodução total ou parcial do trabalho sem autorização da universidade, do autor e do orientador.

\section{Fillipe Machado Pinto Napolitano}

Graduou-se em Engenharia de Telemática pelo Instituto Militar de Engenharia - IME, em novembro de 2004. Área de interesse acadêmico: Engenharia de Software, mais especificamente em Engenharia de Requisitos. Atualmente trabalha no Centro Tecnológico do Exército, como adjunto a Divisão de Tecnologia da Informação.

Ficha Catalográfica

Napolitano, Fillipe Machado Pinto

Uma estratégia baseada em simulação para validação de modelos em $i^{*} /$ Fillipe Machado Pinto Napolitano; orientador: Julio Cesar Sampaio do Prado Leite - 2009

$164 f ; 30 \mathrm{~cm}$

Dissertação (Mestrado em Informática) - Pontifícia Universidade Católica do Rio de Janeiro, Rio de Janeiro, 2009.

Inclui bibliografia.

1. Informática - Teses. 2. Engenharia de Requisitos. 3. Orientação a metas. 4. Framework i*. 5. Validação. 6. Simulação I. Leite, Julio César Sampaio do Prado. II. Pontifícia Universidade Católica do Rio de Janeiro. Departamento de Informática. III. Título. 


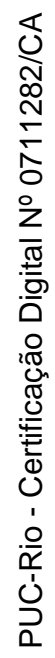

Este trabalho é dedicado à minha família. 


\section{Agradecimentos}

Ao meu orientador, professor Julio Cesar Sampaio do Prado Leite, pela confiança depositada em mim, pela paciência e por todas as orientações durante este trabalho.

À professora Simone Diniz Junqueira Barbosa, pela disposição e importante colaboração durante meus estudos.

À minha querida esposa Andreia, pelo carinho e compreensão nos momentos difíceis.

Aos amigos do grupo de Engenharia de Requisitos, pelas valiosas contribuições a este trabalho.

Aos amigos da Divisão de Tecnologia da Informação - CTEx, pelos ensinamentos e incentivo. 


\section{Resumo}

Napolitano, Fillipe Machado Pinto; Leite, Julio Cesar Sampaio do Prado. Uma Estratégia Baseada em Simulação para Validação de Modelos em i*. Rio de Janeiro, 2009. 164p. Dissertação de Mestrado - Departamento de Informática, Pontifícia Universidade Católica do Rio de Janeiro.

O entendimento da organização antes de iniciar o desenvolvimento dos sistemas organizacionais tem se mostrado bastante eficaz na elicitação de requisitos. Neste contexto, a utilização dos conceitos de intencionalidade através do Framework $i^{*}$ tem sido amplamente empregada por pesquisadores e em algumas empresas. Porém, o uso do framework $i^{*}$ para a modelagem da intencionalidade organizacional carrega a complexidade intrínseca dos modelos. Sendo assim, este trabalho tem como principal objetivo elaborar uma estratégia baseada em simulação para ajudar o engenheiro de requisitos a validar os modelos em $\mathrm{i}^{*}$ elicitados junto aos interessados, sem que os mesmos precisem absorver a complexidade inerente a esses modelos. São apresentados também os resultados obtidos com a aplicação desta estratégia em um estudo de caso.

\section{Palavras-chave}

Engenharia de requisitos; framework i*; validação; simulação 


\section{Abstract}

Napolitano, Fillipe Machado Pinto; Leite, Julio Cesar Sampaio do Prado (Advisor). A Simulation-Based Validation Strategy to i* Models. Rio de Janeiro, 2009. 164p. MSc. Dissertation - Departamento de Informática, Pontifícia Universidade Católica do Rio de Janeiro.

The understanding of the organization before starting the development of organizational systems has been very effective in requirements elicitation. In this context, the use of intentionality concepts through the $\mathrm{i} *$ framework has been widely used by researchers and by some companies. However, the use of $\mathrm{i}^{*}$ framework to modeling organizational intentionality brings the inherent complexity of the models. Thus, this work has the main objective to develop a simulation-based strategy to help the requirements engineer to validate the $i^{*}$ models elicitated with the stakeholders, without absorbing the inherent complexity of these models. We also present the results of the strategy implementation in a case study.

\section{Keywords}

Requirements engineering; i* framework; validation; simulation 


\section{Sumário}

1 Introdução 17

1.1. Motivação 17

1.2. Objetivos 18

$\begin{array}{lr}\text { 1.3. Conceitos } & 19\end{array}$

1.3.1. Requisitos Funcionais 19

1.3.2. Requisitos Não Funcionais 19

1.3.3. Ação Concreta x Ação Flexível 19

1.3.4. Léxico Estendido da Linguagem (LEL) 20

1.3.5. Cenários 20

1.3.6. Grafos NFR 20

1.4. Organização da Dissertação 21

2 O Framework de Modelagem i ${ }^{*} 22$

2.1. Visão geral do framework $i^{*}$

2.2. O Modelo SD - Strategic Dependency 23

2.3. O Modelo SR - Strategic Rationale 26

2.4. Extensões do framework $i^{*} \quad 29$

2.4.1. O Modelo SA - Strategic Actor 29

2.4.2. Situação de Dependência Estratégica - SDsituations 32

2.4.3. Painel de Intencionalidade - Diagrama IP 34

3 Estratégia de Validação Baseada em Simulação 37

3.1. Visão Geral da Estratégia 37

3.2. Construir Modelos $i^{\star} \quad 39$

3.2.1. Elicitar as Metas dos Atores 41

3.2.1.1. Preparar o Léxico Estendido da Linguagem 41

3.2.1.2. Definir Metas dos Agentes Vindas do Léxico - AGFL 42

3.2.1.3. Refinar as Metas 44

3.2.2. Identificar as Situações de Dependência Estratégica 45

3.2.2.1. Distinguir SDsituations 45 
3.2.2.2. Reconhecer Interdependências entre SDsituations 46

3.2.2.3. Construir Diagramas de SDsituations 46

3.2.3. Modelar as Metas dos Atores 47

3.2.3.1. Identificar Agentes, Posições e Papéis 47

3.2.3.2. Criar os Painéis de Intencionalidade 48

3.2.4. Modelar a Racionalização das Metas dos Atores 49

3.2.4.1. Construir Modelos SD 49

3.2.4.2. Construir Modelos SR 50

3.2.5. Especificar as SDsituations $\quad 51$

3.2.6. Analisar os Modelos SD e SR 54

3.3. Transformar 55

3.3.1. Heurísticas de Transformação 55

3.3.2. Tratando Restrições do Tipo Metas Flexíveis 57

3.4. Validar por Simulação 61

3.4.1. Heurísticas de Validação por Simulação 63

4 Estudo de Caso 66

4.1. SimulES 66

4.1.1. Cartão de Projetos $\quad 67$

4.1.2. Tabuleiros 68

4.1.3. Cartas 68

4.1.4. Artefatos 69

4.2. Aplicação da Estratégia no Projeto SimulES 70

4.2.1. Elicitar as Metas dos Atores 70

4.2.1.1. Preparar o LEL - Léxico Estendido da Linguagem 70

4.2.1.2. Definir AGFL - Metas dos Agentes Vindas do Léxico 70

4.2.2. Identificar as Situações de Dependência Estratégica 93

4.2.3. Modelar as Metas dos Atores 96

4.2.4. Modelar a Racionalização das Metas dos Agentes 103

4.2.5. Especificar as SDsituations 114

4.3. Transformar 118

4.4. Validar por Simulação 125

4.4.1. Tratando os Resultados Obtidos 127 
5 Conclusões

5.1. Análise de Trabalhos Relacionados 153

5.1.1. i Diagnoses: A Quality Process for Building i Models [28] 153

5.1.2. A Survey of Good Practices and Misuses for Modelling with $\mathrm{i}^{*}$ Framework [29]

5.1.3. A Method for the Definition of Metrics over $\mathrm{i}^{*}$ Models [30] 156

5.1.4. Comparação com a Literatura Apresentada 158

5.2. Avaliação dos Resultados Obtidos 158

5.3. Contribuições 159

5.4. Trabalhos Futuros 160

6 Referências Bibliográficas 162 


\section{Lista de Figuras}

Figura 2.1 - Tipos de dependências entre atores no modelo SD. 24

Figura 2.2 - Exemplo de um modelo SD. Retirado de [2]. 25

Figura 2.3 - Decomposição de tarefas (parte). Baseado em [2]. 27

Figura 2.4 - Elos meios-fim (parte). Baseado em [2]. 28

Figura 2.5 - Exemplo de Modelo SR. Retirado de [2]. 29

Figura 2.6 - Exemplo de um modelo SA. 31

Figura 2.7 - Exemplo de interdependências entre SDsituations.

Adaptado de [3].

Figura 2.8 - Diagrama de classes de uma SDsituation.

Traduzido de [2].

Figura 2.9 - Exemplo de um painel de intencionalidade [2]. 36

Figura 3.1 - SADT da estratégia de validação de modelos em i*. 38

Figura 3.2 - Etapas do método ERi ${ }^{\star}$ C. Adaptado de [2]. 40

Figura 3.3 - Exemplo de símbolos do LEL [19]. 42

Figura 3.4 - Exemplo de template para ações

concretas (sujeito e objeto) [2].

Figura 3.5 - Exemplo de template para ações

flexíveis (sujeito e objeto) [2].

Figura 3.6 - Metas agrupadas por ator cronologicamente organizadas [2].

Figura 3.7 - Metas concretas e flexíveis de caixa

organizadas por SDsituations.

Figura 3.8 - Diagrama de SDsituations - Controle de Caixa de Restaurante [2].

Figura 3.9 - Diagrama SA - Controle do Caixa de Restaurante $\quad 47$

Figura 3.10 - Diagrama IP - Atendimento da Mesa [2]. 48

Figura 3.11 - Modelo SD para Atendimento da Mesa [2]. 50

Figura 3.12 - Modelo SR da situação Atendimento de

Mesa. Retirado de [2].

Figura 3.13 - Modelo de Cenário. Traduzido de [13]. 
Figura 3.14 - Exemplo de representação em cenário. 54

Figura 3.15 - Exemplo de grafo NFR. 58

Figura 3.16 - Exemplo de metas flexíveis conflitantes. 59

Figura 3.17 - Metas flexíveis inseridas nos cenários da ferramenta. $\quad 61$

Figura 3.18 - Simulação do cenário Atendimento da Mesa no UCEd. 63

Figura 3.19 - Escolha de um episódio fora da ordem inicial adotada. 64

Figura 4.1 - Cartão de Projeto do SimulES [25].

Figura 4.2 - Tabuleiro individual com engenheiros [26]. 68

Figura 4.3 - Exemplos de cartas [25]. 69

Figura 4.4 - Artefatos com e sem defeito [25]. 69

Figura 4.5 - LEL dos símbolos do tipo sujeito. 71

Figura 4.6 - Template preenchido com metas dos símbolos do tipo sujeito. 73

Figura 4.7 - LEL dos símbolos do tipo objeto. 77

Figura 4.8 - Template com metas concretas dos símbolos do tipo objeto. 82

Figura 4.9 - Template com metas flexíveis dos símbolos do tipo objeto. 83

Figura 4.10 - LEL dos símbolos do tipo verbo. 85

Figura 4.11 - Template com metas dos símbolos do tipo verbo. 89

Figura 4.12 - LEL dos símbolos do tipo estado. 90

Figura 4.13 - Template com metas dos símbolos do tipo estado. 92

Figura 4.14 - Metas agrupadas por ator e cronologicamente organizadas. 93

Figura 4.15 - Metas organizadas em SDsituations. 95

Figura 4.16 - Diagrama de SDsituations do SimulES. 96

Figura 4.17 - O modelo SA do SimulES. 97

Figura 4.18 - Diagrama IP - Joga Rodada de Início. 97

Figura 4.19 - Diagramas IP - Joga Rodada de Ações. 98

Figura 4.20 - Diagramas IP - Construção de Artefatos

e Inspeção de Artefatos.

Figura 4.21 - Diagramas IP: Correção de Artefatos

e Integração de Artefatos em Módulo. 
Figura 4.22 - Diagrama IP: Joga Rodada de Conceitos.

Figura 4.23 - Diagramas IP - Tratamento de Problema e Submissão de Produto.

Figura 4.24 - Modelo SD - Joga Rodada de Início. 103

Figura 4.25 - Modelo SR - Joga Rodada de Início. 104

Figura 4.26 - Modelo SD - Joga Rodada de Ações. 104

Figura 4.27 - Modelo SR - Joga Rodada de Ações. 105

Figura 4.28 - Modelo SD - Construção de Artefato. 106

Figura 4.29 - Modelo SR - Construção de Artefato. 106

Figura 4.30 - Modelo SD - Inspeção de Artefato. 107

Figura 4.31 - Modelo SR - Inspeção de Artefato. 107

Figura 4.32 - Modelo SD - Correção de Artefato. 108

Figura 4.33 - Modelo SR - Correção de Artefato. 108

Figura 4.34 - Modelo SD - Integração de Artefato em Módulo. 109

Figura 4.35 - Modelo SR - Integração de Artefato em Módulo. 109

Figura 4.36 - Modelo SD - Joga Rodada de Conceitos. 110

Figura 4.37 - Modelo SR - Joga Rodada de Conceitos. 111

Figura 4.38 - Modelo SD - Tratamento de Problema. 112

Figura 4.39 - Modelo SR - Tratamento de Problema. 112

Figura 4.40 - Modelo SD - Submissão de Produto. 113

Figura 4.41 - Modelo SR - Submissão de Produto. 113

Figura 4.42 - SDsituation Joga Rodada de Início (descrição). 114

Figura 4.43 - SDsituation Joga Rodada de Ações (descrição). 114

Figura 4.44 - SDsituation Construção de Artefato (descrição). 115

Figura 4.45 - SDsituation Inspeção de Artefato (descrição). 115

Figura 4.46 - SDsituation Correção de Artefato (descrição). 116

Figura 4.47 - SDsituation Integração de Artefatos em

Módulo (descrição). 116

Figura 4.48 - SDsituation Joga Rodada de Conceitos (descrição). 117

Figura 4.49 - SDsituation Tratamento de Problema (descrição). 117

Figura 4.50 - SDsituation Submissão de Produto (descrição). 118

Figura 4.51 - Grafo NFR da meta flexível Qualidade [artefato]. 119

Figura 4.52 - Grafo NFR da meta flexível Qualidade [projeto]. 119 
Figura 4.53 - Cenário Joga Rodada de Início (transformado). 120

Figura 4.54 - Cenário Joga Rodada de Ações (transformado). 121

Figura 4.55 - Cenário Construção de Artefato (transformado). 121

Figura 4.56 - Cenário Inspeção de Artefato (transformado). 122

Figura 4.57 - Cenário Correção de Artefato (transformado). 122

Figura 4.58 - Cenário Integração de Artefato em

Módulo (transformado). 123

Figura 4.59 - Cenário Joga Rodada de Conceitos (transformado). 123

Figura 4.60 - Cenário Tratamento de Problema (transformado). 124

Figura 4.61 - Cenário Submissão de Produto (transformado). 124

Figura 4.62 - Alterações realizadas pelo Interessado 1

durante a simulação.

126

Figura 4.63 - Alterações realizadas pelo Interessado 2

durante a simulação.

126

Figura 4.64 - Alterações realizadas pelo Interessado 3

durante a simulação.

Figura 4.65 - Alterações resultantes realizadas pelos Interessados. 127

Figura 4.66 - Template da meta flexível Boa [estratégia] (parcial). 130

Figura 4.67 - Símbolos impactados pela meta flexível

Boa [estratégia].

Figura 4.68. Metas refinadas com a adição da meta

flexível Boa [estratégia].

Figura 4.69. Diagramas IP Joga Rodada de Ações e Tratamento de Problema com a adição da meta flexível Boa [estratégia].

Figura 4.70. Diagramas IP Inspeção de Artefato e Correção

de Artefato com a adição da meta flexível Boa [estratégia].

Figura 4.71. Diagramas IP Joga Rodada de Conceitos com

a adição da meta flexível Boa [estratégia].

Figura 4.72. Diagrama SR Joga Rodada de Ações modificado.

Figura 4.73. Diagrama SR Inspeção de Artefato modificado.

Figura 4.74. Diagrama SR Correção de Artefato modificado.

Figura 4.75. Diagrama SR Joga Rodada de Conceitos modificado. 139

Figura 4.76. Diagrama SR Tratamento de Problema modificado. 
Figura 4.77 - Descrição da SDsituation Joga Rodada de Início antes e depois da correção.

Figura 4.78 - Descrição da SDsituation Joga Rodada de Ações antes e depois da correção.

Figura 4.79 - Descrição da SDsituation Construção de Artefato antes e depois da correção.

Figura 4.80 - Descrição da SDsituation Inspeção de Artefato antes e depois da correção.

Figura 4.81 - Descrição da SDsituation Correção de Artefato antes e depois da correção.

Figura 4.82 - Descrição da SDsituation Joga Rodada de Conceitos antes e depois da correção.

Figura 4.83 - Descrição da SDsituation Tratamento de Problemas antes e depois da correção.

Figura 4.84 - Grafo NFR para Boa [estratégia].

Figura 4.85 - Novo cenário Joga Rodada de Inicio (transformado). 148

Figura 4.86 - Novo cenário Joga Rodada de Ações (transformado). 148

Figura 4.87 - Novo cenário Construção de Artefato (transformado). 149

Figura 4.88 - Novo cenário Inspeção de Artefato (transformado). 149

Figura 4.89 - Novo cenário Correção de Artefato (transformado). 150

Figura 4.90 - Novo cenário Joga Rodada de

Conceitos (transformado).

Figura 4.91 - Novo cenário Tratamento de

Problema (transformado). 


\section{Lista de tabelas}

Tabela 3.1 - Regras gerais para definição de símbolos [18].

Tabela 3.2 - Regras para definição de dependências estratégicas. $\quad 49$ 